\title{
Large scale genome-wide association and LDLA mapping study identifies QTLs for boar taint and related sex steroids
}

\author{
Eli Grindflek ${ }^{1 *}$, Sigbjørn Lien ${ }^{2,3}$, Hanne Hamland ${ }^{1,3}$, Marianne HS Hansen ${ }^{1,3}$, Matthew Kent ${ }^{2,3}$, Maren van Son ${ }^{1}$ and \\ Theo HE Meuwissen ${ }^{2}$
}

\begin{abstract}
Background: Boar taint is observed in a high proportion of uncastrated male pigs and is characterized by an unpleasant odor/flavor in cooked meat, primarily caused by elevated levels of androstenone and skatole.

Androstenone is a steroid produced in the testis in parallel with biosynthesis of other sex steroids like testosterone and estrogens. This represents a challenge when performing selection against androstenone in breeding programs, without simultaneously decreasing levels of other steroids. The aim of this study was to use high-density genome wide association (GWA) in combination with linkage disequilibrium-linkage analysis (LDLA) to identify quantitative trait loci (QTL) associated with boar taint compounds and related sex steroids in commercial Landrace $(\mathrm{n}=1,251)$ and Duroc $(\mathrm{n}=918)$ breeds.

Results: Altogether, 14 genome wide significant (GWS) QTL regions for androstenone in subcutaneous fat were obtained from the LDLA study in Landrace and 14 GWS QTL regions in Duroc. LDLA analysis revealed that 7 of these QTL regions, located on SSC 1, 2, 3, 7 and 15, were obtained in both breeds. All 14 GWS androstenone QTLS in Landrace are also affecting the estrogens at chromosome wise significance (CWS) or GWS levels, while in Duroc, 3 of the 14 QTLs affect androstenone without affecting any of the estrogens. For skatole, 10 and 4 QTLs were GWS in the LDLA analysis for Landrace and Duroc respectively, with 4 of these detected in both breeds. The GWS QTLS for skatole obtained by LDLA are located at SSC 1, 5, 6, 7, 10, 11, 13 and 14 .

Conclusion: This is the first report applying the Porcine $60 \mathrm{~K} \mathrm{SNP}$ array for simultaneous analysis of boar taint compounds and related sex hormones, using both GWA and LDLA approaches. Several QTLs are involved in regulation of androstenone and skatole, and most of the QTLs for androstenone are also affecting the levels of estrogens. Seven QTLs for androstenone were detected in one breed and confirmed in the other, i.e. in an independent sample, although the majority of QTLs are breed specific. Most QTLs for skatole do not negatively affect other sex hormones and should be easier to implement into the breeding scheme.
\end{abstract}

\section{Background}

In spite of the increasing social pressure against it, surgical castration is still commonly performed to eliminate boar taint in male slaughter pigs. However, entire male pigs show better feed consumption, leaner meat percentage and better carcass traits than barrows and are more profitable for swine producers [e.g. 1]. An alternative to castration would therefore be highly advantageous for

\footnotetext{
* Correspondence: eli.grindflek@norsvin.no

'NORSVIN (The Norwegian Pig Breeders Association), PO Box 504, 2304

Hamar, Norway

Full list of author information is available at the end of the article
}

pig breeders worldwide. Boar taint is a characteristic and unpleasant odor and flavor noticeable when cooking meat from some uncastrated male pigs. The taint has been described as being akin to urine and manure [2] and is mainly caused by high levels of androstenone, skatole and/or indole in adipose tissue. The incidence of tainted carcasses varies considerably between breeds and the contribution of androstenone and skatole to boar taint has also been shown to be very different among breeds [e.g. 3]. Heritability of androstenone is high, ranging from 0.25 to $0.88[3,4]$. Androstenone is a natural steroid produced by the Leydig cells of the testis along

\section{Biomed Central}


with other sex steroids like estrogens and testosterone [5], but it is subsequently concentrated and converted in the salivary gland to an active sex pheromone. While estrogens are well known as important female sex hormones, they are also important controllers for the normal function of the adult male reproductive tract and male fertility (reviewed by Hess [6]) and serve as indicator of sexual maturity status in boars $[7,8]$. Therefore to identify genomic regions simultaneously affecting levels of boar taint and fertility related compounds, the sex hormones $17 \beta$ estradiol (one of the main estrogens), estrone sulphate (the reservoir for the active compound of $17 \beta$-estradiol) and testosterone (essential to maintain spermatogenesis and male fertility) [reviewed by 9] were included. Two other compounds, skatole and indole, are produced from the amino acid tryptophan in the colon and are absorbed into the blood stream before being degraded in the liver [10]. While indole is thought to be less important with regards to boar taint [11], skatole is found to have a strong faecal and naphthalene taste and odor [12]. Skatole is shown to have medium/high heritability $(0.37-0.41)$ in Norwegian Landrace and Duroc [3]. Levels of skatole are affected by feeding and recently Rasmussen et al. [13] demonstrated that feed containing chicory root could impact the activity of enzymes involved in skatole metabolism.

Genome scans have been performed for androstenone and skatole in a variety of pig breeds [14-17], however results have been inconsistent. Recent developments in high-throughput genotyping technologies and efficient methods for SNP-discovery using next-generation sequencing technologies have paved the way to create efficient tools for QTL mapping by genome-wide association. A high-density porcine SNP array containing more than 60,000 SNPs has been developed [18], which offers a much higher resolution compared to the previously available porcine SNP chip [19] used in our populations [3]. The aim of this study was to perform a high-density genome wide association (GWA) in combination with linkage disequilibrium-linkage analysis (LDLA) using the $60 \mathrm{~K}$ SNP chip to identify the genomic regions influencing the boar taint compounds androstenone, skatole and indole, as well as the fertility related compounds $17 \beta$-estradiol, estron sulphate and testosterone in the two commercial breeds Duroc and Landrace. Additionally, differentially expressed genes within the QTL regions were detected by combining the current results with the results from Moe et al. [20] and [21]. This identifies genes located within the target regions providing both transcriptional and positional candidate genes for the QTL.

\section{Methods}

\section{Animals and phenotypes}

In total, data from 1,251 purebred Norwegian Landrace and 918 purebred Duroc male pigs tested in NORSVIN's three boar testing stations were included in this study. This includes 92 half sib families with 7 to 24 offspring for Landrace and 70 half sib families with 6 to 27 offspring for Duroc. Pedigree information, including six generations of parents, was available for all individuals. Animals were reared under similar conditions using standard commercial feed, and were sacrificed over a period of 26 months. On average, Landrace and Duroc boars reached slaughter weight $(100 \mathrm{~kg})$ at 143 and 156 days respectively, and were slaughtered in average 15 days later. The reason for this is that we had to wait for final EBV estimation and selection of the AI boars. Blood samples were collected immediately before slaughter for plasma suspension and DNA extraction, while subcutaneous adipose tissue samples for androstenone measurement were collected from the neck at slaughter line (20-30 minutes post-mortem). All animals were cared for according to laws, internationally recognized guidelines and regulations controlling experiments with live animals in Norway (The Animal Protection Act of December 20th, 1974, and the Animal Protection Ordinance Concerning Experiments with Animals of January 15th, 1996); according to the rules given by Norwegian Animal Research Authority.

Levels of androstenone were measured in both subcutaneous fat and plasma and in both cases the concentration of androstenone (ppm) was determined using a modified time-resolved fluoroimmunoassay [22], employing an antibody produced by Andresen [23]. Skatole and indole levels in adipose tissue were analyzed using high performance liquid chromatography [24]. Plasma levels of testosterone were measured using a radioimmunoassay (Orion Diagnostica, Espoo, Finland) and displayed intra- and total-assay coefficients of variation (CVs) were $7 \%$ and $9 \%$, respectively. Plasma levels of $17 \beta$-estradiol were measured using a fluoroimmunoassay (Perkin Elmer, Turku, Finland) producing intraand total-assay CVs of $3 \%$ and $7 \%$, respectively. Plasma levels of estrone sulphate were measured with a radioimmunoassay (Diagnostic System Laboratories, Inc., Webster, TX, USA) producing intra- and total-assay CVs of $5 \%$ and $7 \%$, respectively. All chemical compounds are reported in parts-per-million (ppm). All the phenotypic data were log-transformed in order to make them approximately normally distributed.

\section{DNA extraction, genotyping, quality control and map construction}

DNA was extracted from porcine blood, leukocytes or semen using the MagAttract DNA Blood Midi M48 protocol on the Bio-Robot M48 (Qiagen, Hilden, Germany). DNA concentration and quality were assessed using a NanoDrop ND-1000 spectrophotometer (NanoDrop Technologies, DE, USA) and a Victor ${ }^{3}$ Multilabel 
Counter (model 1420, Turku, Finland) using PicoGreen reagent (Molecular Probes, OR, USA). The DNA quality was considered to be acceptable with lower and upper limits of 25 and $75 \mathrm{ng} / \mu \mathrm{l}$, respectively, and with 260:280 and 260:230 ratios around 1.8. Thereafter samples were normalized to $50 \mathrm{ng} / \mu \mathrm{l}$ in 96-well plates. Genotyping of the $60 \mathrm{~K}$ porcine SNP array was performed using the iScan platform (Illumina, San Diego, CA, USA) according to manufacturer's instructions. Clustering and genotype calling were performed using the genotyping module in the Genome Studio software (Illumina, San Diego, CA, USA). In total, the 1,251 Landrace and 918 Duroc boars were genotyped for 60,451 SNPs. Genotypes were included in analysis if SNP markers passed a quality threshold of having a minor allele frequency $(\mathrm{MAF})>0.01$, call frequency $>0.10$, and Parent-Child Mendelian errors $<0.025$. Samples were included in analysis if their call rate was $>75 \%$, although the average call rate was $99.4 \%$ with a standard deviation of $1.8 \%$. Based on data from a previous study, samples from animals displaying excessive pedigree errors were not included in this analysis. After quality control 1,155 boars and 86 sires were available for analysis in Landrace together with 840 boars and 68 sires in Duroc. In total 51,943 SNP markers (86\%) passed the quality threshold filtering. Due to being non-informative 7117 SNPs were removed. Additionally, 346 SNPs were removed since they were not able to place on any of the chromosomes using two-point analysis in a modified version of the CriMap software package [25]. The order of markers was determined based on the porcine sequence Build 9 (Sanger Institute) followed by multipoint linkage analyses using CriMap [25]. Recombination units were then transformed to map distances using the Haldane mapping function, and the suggested positions from Sscrofa9 were modified if required. In total 44,480 of the high quality SNP markers were placed on chromosomal locations on Sscrofa9, 38,396 on the Landrace map and 36,869 on the Duroc map. Number of SNP markers per chromosome and length of the chromosomes (bp and $\mathrm{cM}$ ) are shown in Additional file 1 .

\section{Statistical analyses}

Statistical analyses were performed separately for the two breeds. Preliminary estimates of the phenotypes were found to have a skewed distribution and were consequently log-transformed. Furthermore some animals had phenotypic levels below the detection limits of the chemo analytical methods used and were recorded as zero. To avoid losing information caused by log-transformation, the detection limits were added to all phenotypes before transformation. The detection limits were $0.05 \mathrm{ppm}$ for androstenone, $0.01 \mathrm{ppm}$ for skatole and indole, $0.5 \mathrm{ppm}$ for testosterone and estron sulphate, and $0.04 \mathrm{ppm}$ for $17 \beta$-estradiol. GWA is considered to be more powerful than linkage analysis for detecting the effects of common alleles with small effects but is less powerful when traits have a complex genetic determination, including epistasis. The GWA method uses linkage disequilibrium information and assumes all markers to be independent of each other, thereby ignoring genetic linkage between markers. GWA is expected to reveal more false positive results because single marker linkage disequilibria are very variable. Therefore, the data was simultaneously analyzed with LDLA, combining the linkage disequilibrium and linkage analysis [26].

\section{Genome-wide association study (GWAS)}

A GWAS was conducted using a statistical model including fixed effect corrections for; test-station, age at entering the test, number of days in test, number of litter mates born alive, and the random effects; a polygenic effect and an additive SNP effect. The polygenic effect was included to account for the family structure that was present in the data and was assumed to have a covariance matrix proportional to the pedigree based relationship matrix. The additive SNP effect was also assumed a random effect, and was obtained by regressing the phenotypes on to the number of ' 1 ' alleles in the SNP genotypes, i.e. for the SNP genotypes ' 00 ', ' 0 1 ', and ' 11 ' the covariate of the regression was 0,1 and 2 , respectively, where arbitrarily one of the SNP alleles is called ' 1 ' and the other ' 0 '.

A log-likelihood ratio test-statistic was calculated as LnLikratio $=$ LnLik $_{\text {model incl. SNP }}-$ LnLik $_{\text {model excl. SNP, }}$ where LnLik $k_{\text {model incl. SNP }}$ is the REML log-likelihood [27] of the model describe above and LnLik $k_{\text {model excl. SNP }}$ is the REML log-likelihood of the same model, except that the SNP effect was excluded from the analysis. Under the null-hypothesis of no SNP effect, $2 *$ LnLikratio was assumed to follow a chi-squared distribution with one degree of freedom. Chromosome-wide P-values (CWS) were obtained using the approach described by Piepho [28]. Genome-wide P-values (GWS) were obtained by multiplying the CWS by 18 , i.e. by the number of porcine autosomal chromosomes analyzed. We considered the SNP/QTL as significant if $\mathrm{P}<0.05$.

\section{Linkage disequilibrium/linkage analysis (LDLA)}

The statistical model used for the combined linkage disequilibrium and linkage analysis (LDLA) was identical to that of GWAS except that a haplotype effect was fitted instead of a SNP effect. LDLA followed approximately the approach of Meuwissen and Goddard [26], except that haplotypes were either assumed to be completely correlated or uncorrelated, instead of fitting a more differentiating IBD matrix G. Based on experiences with IBD matrix calculations it was decided that if the sum of the number of identical SNP alleles equal to the left 
and to the right of the putative QTL position before a non-equal allele occurred was $\geq 10$, then a haplotype pair was considered identical. Otherwise, the two haplotypes were considered to be different, and uncorrelated. LnLikratio, CWS and GWS were calculated in the same way as for GWAS.

\section{Candidate genes and differential expressed genes within QTL regions}

Candidate genes within the QTL regions were detected using the porcine Ensembl data base (http://www. ensembl.org/Sus_scrofa/Info/Index) (Build 9). In order to obtain additional candidate genes in comparative genomic regions the UCSC genome browser (http://genome.ucsc.edu/index.html) and the NCBI database (Build37) were applied. To find differentially expressed genes within the significant QTL regions, the results from the current QTL study was compared with the significant differentially expressed genes obtained using the same animal material $[20,21]$. The results were imported and combined in R v.2.9.2 [29].

\section{Results}

Descriptive data (number of animals, overall means and standard deviations of log-transformed data, and max/ min values) for this animal material have been presented earlier by Grindflek et al. [3]. Here we present QTL results from the seven traits; androstenone in subcutaneous fat (AndroF), androstenone in plasma (AndroP), skatole (Skat), indole (Indo), testosterone (Testo), 17ßestradiol (Ediol) and estronsulphate (Esulph).

\section{Androstenone and other sex steroids}

According to the threshold values described in Material and Methods we detected 14 GWS QTL regions for AndroF in Landrace, and 14 GWS QTL regions in Duroc using LDLA. Seven of these QTL regions were detected in both breeds, in every case they were significant $(\mathrm{P}<0.05)$ at the GWS level in one breed and at least CWS in the other $(\mathrm{P}<0.05)$. The common QTLs were located at SSC 1, 2, 3, 7 and 15. Results from the GWAS revealed 10 QTL regions for AndroF that were GWS in Landrace, 7 of these were located within QTL regions detected by LDLA. In Duroc, 11 QTLs were detected with GWAS, 10 falling within QTL regions identified with LDLA. For AndroP, 14 and 8 GWS QTLs were detected in Landrace and Duroc (respectively) using LDLA (Table 1), with 7 and 5 of them confirmed using GWAS, respectively (Figure 1 and 2). When comparing the QTL regions detected by LDLA, we found that 12 QTLs are significant for both AndroF and AndroP in Landrace, and 4 in Duroc. Comparing QTL regions at the CWS level, a greater number of QTL regions are shared between the AndroF and
Table 1 The genome significant QTLs (from LDLA) for androstenone in fat (AndroF)

\begin{tabular}{|c|c|c|c|c|c|c|}
\hline SSC & QTL & Breed & LnLikratio & CWS_P** & GWS_P** & Mb_Conf.Int. ${ }^{\$}$ \\
\hline 1 & $1 \mathrm{a}$ & $L^{*} / D^{*}$ & $11.57 / 5.87$ & 0.0014 & 0.025 & $15.2-16.3$ \\
\hline 1 & $1 b$ & $D^{*}$ & 11.67 & 0.0006 & 0.011 & $33.0-33.4$ \\
\hline 1 & $1 c$ & $L^{*}$ & 15.07 & 0.0002 & 0.004 & $44.3-44.9$ \\
\hline 1 & $1 d$ & $L^{*}$ & 11.73 & 0.0013 & 0.023 & $53.5-54.3$ \\
\hline 2 & $2 \mathrm{a}$ & $L^{*} / D^{*}$ & $12.73 / 9.06$ & 0.0016 & 0.029 & $29.9-37.0$ \\
\hline 2 & $2 b$ & $L^{*} / D$ & $9.11 / 21.12$ & $<0.0001$ & $<0.001$ & $101.4-101.7$ \\
\hline 2 & $2 c$ & $L^{*}$ & 12.32 & 0.0020 & 0.036 & $132.8-135.9$ \\
\hline 3 & 3a & $L^{*} / D^{*}$ & $14.19 / 11.07$ & 0.0003 & 0.005 & $32.2-53.6$ \\
\hline 3 & $3 b$ & $L^{*}$ & 18.73 & $<0.0001$ & $<0.001$ & $107.0-107.1$ \\
\hline 4 & 4 & $\mathrm{D}$ & 17.52 & $<0.0001$ & $<0.001$ & $47.5-48.0$ \\
\hline 5 & $5 a$ & D & 20.74 & $<0.0001$ & $<0.001$ & $20.4-22.2$ \\
\hline 5 & $5 b$ & $L^{*}$ & 10.23 & 0.0014 & 0.025 & $32.4-34.9$ \\
\hline 6 & 6 & $D^{*}$ & 9.79 & 0.0018 & 0.032 & $118.8-119.2$ \\
\hline 7 & $7 a$ & $L^{*} / D^{*}$ & $7.37 / 9.10$ & 0.0010 & 0.018 & $34.6-40.2$ \\
\hline 7 & $7 b$ & $L / D^{*}$ & $4.59 / 12.36$ & 0.0006 & 0.011 & $62.5-65.0$ \\
\hline 7 & 7c & $D^{*}$ & 11.80 & 0.0008 & 0.014 & $126.9-127.5$ \\
\hline 9 & 9 & $L^{*}$ & 12.01 & 0.0005 & 0.009 & $7.5-8.0$ \\
\hline 10 & 10 & $D^{*}$ & 12.55 & 0.0004 & 0.007 & $35.0-37.0$ \\
\hline 11 & $11 a$ & $D^{*}$ & 10.67 & 0.0011 & 0.020 & $11.8-16.7$ \\
\hline 11 & $11 \mathrm{~b}$ & $L^{*}$ & 12.31 & 0.0005 & 0.009 & $64.2-64.5$ \\
\hline 13 & $13 a$ & $D^{*}$ & 30.61 & $<0.0001$ & $<0.001$ & $19.3-19.6$ \\
\hline 13 & $13 b$ & $L^{*}$ & 13.34 & 0.0003 & 0.005 & $87.8-92.5$ \\
\hline 15 & 15 & $L^{*} / D^{*}$ & $14.80 / 4.85$ & 0.0003 & 0.008 & $42.5-70.7$ \\
\hline 18 & $18 a$ & $L^{*}$ & 10.66 & 0.0011 & 0.020 & $13.4-14.2$ \\
\hline 18 & $18 b$ & $L^{*}$ & 12.26 & 0.0005 & 0.009 & $39.7-40.4$ \\
\hline
\end{tabular}

* Additionally at least Chromosome Wide Significant (CWS) for Ediol and/or Esulph

** The highest P-value from the two breeds

$\$$ The boundaries of the $90 \%$-confidence-interval in mega-bases $(\mathrm{Mb})$.

AndroP. All 14 GWS AndroF QTLs in Landrace are also affecting Ediol and/or Esulph on at least a CWS level. In Duroc, 3 of 14 QTLs are affecting AndroF without at least CWS affecting any of the other estrogens. Only 2 GWS QTLs were detected for Testo (SSC10, 28-37 Mb, and SSC16, 21-24 Mb), however these were very convincingly obtained in both breeds using both LDLA and GWAS. For Ediol, 13 GWS QTLs were obtained in the LDLA for Landrace and 6 for Duroc, with the common QTLs between breeds located on SSC 1, 13 and 15. QTL regions for Esulph were generally similar to those detected for Ediol. For Landrace, 10 of the 13 QTLs obtained in Ediol were also at least CWS in Esulph. Notably however, a convincing QTL on SSC3 (44.5-44.6 Mb) was significant (GWS) in Esulph only. In Duroc all QTLs obtained on GWS level were in common for Ediol and Esulph. The fraction of the genetic variance explained by the significant AndroF QTL regions varies between 1 and 6\% (results not shown). The GWS QTLs for AndroF obtained by LDLA are listed in Table 1 . The log-ratio 

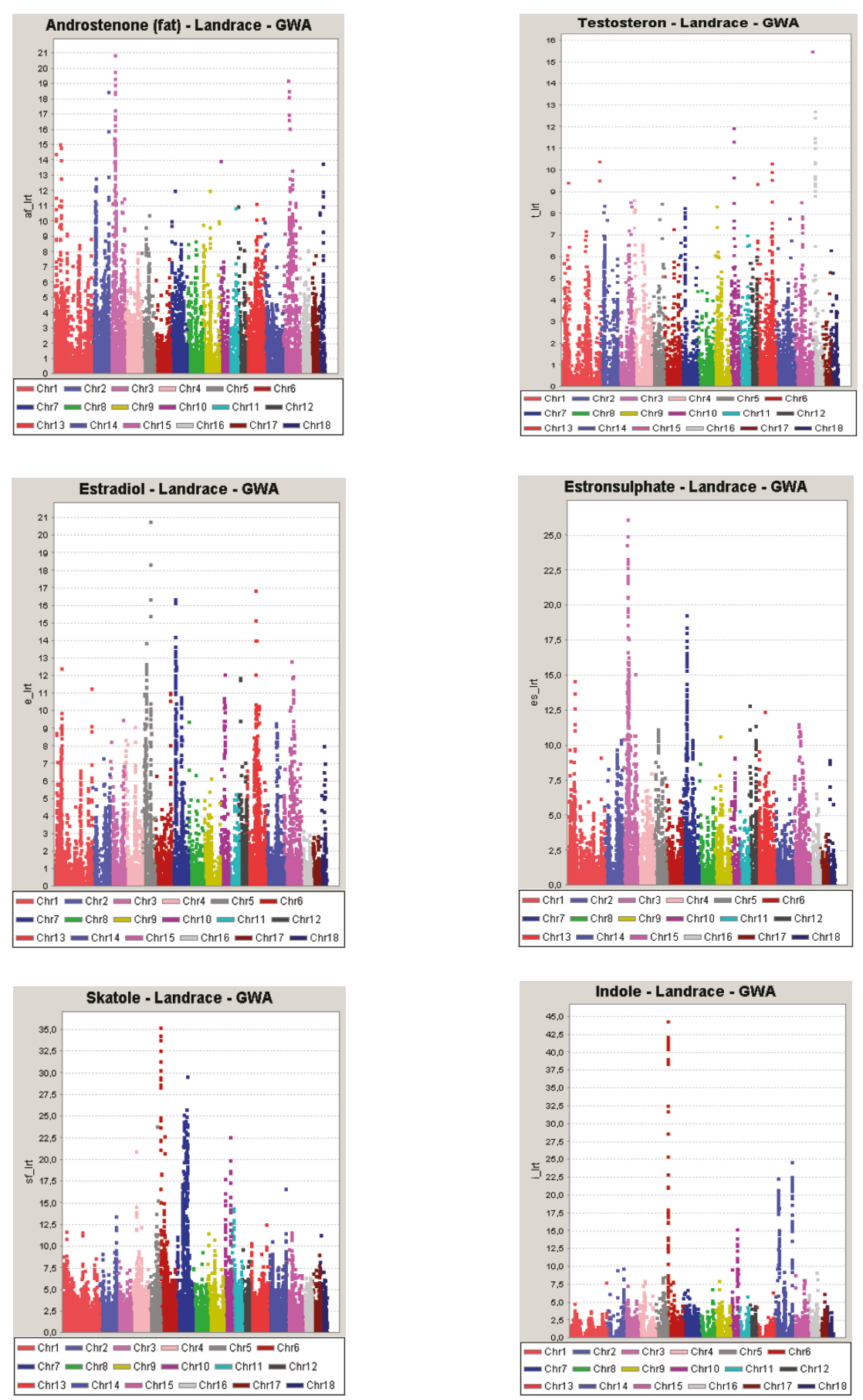

Figure 1 Log-ratio plots for AndroF, Testo, Ediol, Esulph, Skat and Indo from the genome wide association study (GWAS) in Landrace.

plots from GWAS results AndroF, Testo, Ediol and Esulph are shown in Figure 1 and 2 for Landrace and Duroc, respectively. The candidate genes within GWS QTL regions for AndroF are shown in Table 2 and the differentially expressed genes in high/low androstenone boars located within these QTL regions are shown in Table 3.

\section{Skatole and indole}

For skatole, 10 and 4. QTLs were GWS in the LDLA analysis for Landrace and Duroc, respectively, 8 and 4 of them (respectively) were also GWS using GWAS. Four QTLs were obtained in both breeds using the LDLA, on at least CWS level. In 7 of the 10 GWS LDLA QTLs obtained in Landrace, the QTLs were also 

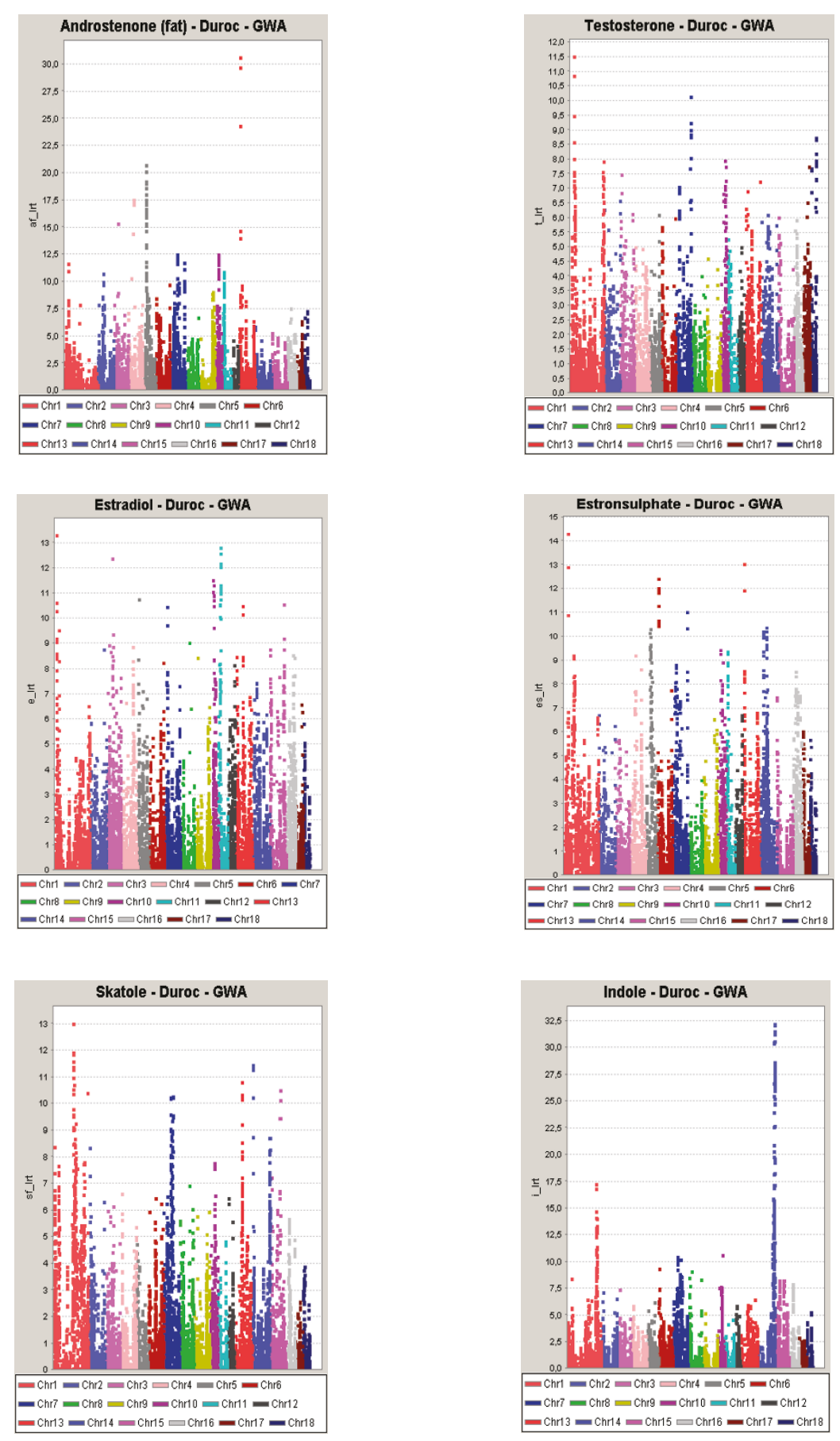

Figure 2 Log-ratio plots for AndroF, Testo, Ediol, Esulph, Skat and Indo from the genome wide association study (GWAS) in Duroc

affecting (at least CWS) the level of Indo. Likewise, 3 of 4 GWS Skat QTLs in Duroc were also significantly affecting Indo. The GWS QTLs for Skat obtained by LDLA are located at SSC 1, 5, 6, 7, 10, 11, 13 and 14, and are all listed in Table 4. Log-ratio plots of GWAS results for Skat and Indo are presented in Figure 1 and 2 for Landrace and Duroc, respectively. The candidate genes within GWS QTL regions for Skat are shown in Table 2.

\section{Discussion}

QTL regions and potential candidate genes

The GWS QTLs for androstenone and skatole obtained by LDLA are divided into the different chromosomes 
Table 2 Candidate genes located within GWS QTL regions and discussed in the paper

\begin{tabular}{|c|c|c|c|c|}
\hline QTL & Positions (Sscrofa9) & Reference & Full name & Gene \\
\hline $2 a$ & $41501759-41519677$ & De Fabiani et al., 2010 & Cytochrome P450, subfamily IIR, polypeptide 1 & CYP2R1 \\
\hline $2 b$ & $10440795-104856025$ & Rodriquez-Agudo et al., 2008 & START domain containing 4, sterol regulated & STARD4 \\
\hline $3 a$ & $39059355-39072049$ & Gumus et al., 2008 & Interleukin Alpha 1 & IL1A \\
\hline $3 a$ & $42095144-42118620$ & Horibata \& Sugimoto, 2010 & START domain containing 7 , sterol regulated & STARD7 \\
\hline $3 a$ & $44005314-44096991$ & Bray et al., 2009 & UDP-glucuronate decarboxylase 1 & UXS1 \\
\hline 4 & $47901914-47937394$ & Amills et al., 2005 & 2,4-alpha-dienoyl-CoA reductase 1 & DECR1 \\
\hline 4 & $47870808-47893791$ & Opperman et al., 1992 & Calbindin 1, 28kDa & CALB1 \\
\hline 5 & hsa12: 56114151-56118525* & Wang et al. 1999 & Retinol dehydrogenase & Rdh5 \\
\hline 5 & hsa12: 57157108- 57181574* & Moeller \& Adamski, 2006 & Hydroxysteroid (17-beta) dehydrogenase 6 homolog & HSD17B6 \\
\hline $7 a$ & $27908299-27911241$ & Crawford et al., 1992 & cytochrome P450, fam. 21, subfam. A, polypeptide 2 iso a & CYP21/CYP21A2 \\
\hline $7 b$ & $64454785-64461043$ & Diaz \& Squires, 2000 & cytochrome P450, family 1, subfamily A, polypeptide 1 & CYP1A1 \\
\hline $7 b$ & hsa15:74630100 - 74660081* & Miller, 1995 & cytochrome P450, family 11 , subfamily A, polypeptide 1 & CYP11A1 \\
\hline 7c & $127029340-127072433$ & Wiebe et al., 2010 & vaccinia related kinase 1 & VRK1 \\
\hline $7 a^{5}$ & $64280270-64285232$ & Lanza and Yost, 2001 & cytochrome P450, family 1 , subfamily A, polypeptide 2 & CYP1A2 \\
\hline 13 & $19112438-19141042$ & Bahn et al., 2005 & solute carrier family 22, member 14 & SLC22A14 \\
\hline 13 & $19146069-19153361$ & Bahn et al., 2005 & solute carrier family 22, member 13 & SLC22A13 \\
\hline 14 & $148132846-148145308$ & Babol et al., 1998 & cytochrome P450, family 2, subfamily E, polypeptide 1 & CYP2E1 \\
\hline 15 & $64064865-64074555$ & Christoffersen et al., 2010 & Glucagon & GCG \\
\hline 15 & hsa8: $38000226-38008783^{*}$ & Christenson \& Strauss, 2000 & Steroidogenic acute regulatory protein & STAR \\
\hline
\end{tabular}

* Gives the comparative human genomic positions on Build37 for the genes not annotated in Sscrofa9.

and discussed below. We chose to focus on the LDLA results since this method is considered to be most robust. Moreover, the LDLA likelihood profiles were smoother than those from GWA which facilitated the positioning of the QTLs. The QTLs detected by LDLA are, however, highly supported by the GWAS results obtained in the study (Figures 1 and 2). Candidate genes located within QTL regions and discussed in the paper are all listed with the full name in Table 2.

\section{SSC1}

\section{Androstenone}

Four putative QTLs were detected for AndroF in Landrace and/or Duroc on SSC1 (positions shown in Table 1). Recently on SSC1, three QTLs were also detected in a Dutch composite Duroc-line [17] and three QTLs in French Large White [30], but all in different regions. No obvious candidate genes were observed in any of the regions detected in our study, however a non-annotated gene transcript (C6orf211) located within QTL1a was previously found to be differentially expressed in Duroc boars with extreme high/low androstenone levels, and the gene GPD1L located within QTL1c was up-regulated in high androstenone Landrace boars [20] (Table 3).

\section{Skatole}

One putative QTL for skatole was detected on SSC1 position 165.5-170.1 Mb (Table 4). This QTL did not overlap with either of the two recently detected QTL positions on SSC1 for skatole in French Large White [30]. The peak positions in French Large-White are
109.5 and $288.75 \mathrm{Mb}$, quite far from our QTL at 165$170 \mathrm{Mb}$, and are most likely two different QTLs not segregating in our Landrace and Duroc populations.

\section{SSC2}

Two regions on SSC2 (29.9-37.0 Mb; QTL2a, and 101.4101.7 Mb; QTL2b) are affecting (GWS) AndroF, AndroP, and one or two of the estrogens in both breeds. QTL2a is additionally CWS for Testo in Landrace. A Landrace specific QTL (2c) in region 132.8-135.9 Mb was found to affect all traits included in this study (at least CWS level). Our QTL2a concurs with a wide QTL region for androstenone detected between markers SW240 (18 Mb) and S0226 (48 Mb) in a Large White $\times$ Meishan cross [15]. A large number of genes are embraced by this region including GAS2 (Table 3) which was previously found to be differentially expressed in testis from Landrace boars with extreme high/low levels of androstenone [20]. One obvious candidate in the region is the CYP2R1 gene (Table 2) which encodes a member of the cytochrome P450 superfamily of enzymes known to catalyze many reactions involved in synthesis of cholesterol, steroids and other lipids (e.g. reviewed by De Fabiani et al. [31]). No QTLs for androstenone have previously been reported close to our QTL2b. Despite QTL2b presenting a narrow QTL region we found several candidate genes that may be involved in processes of androstenone metabolism. One of them is the STARD4 gene which plays an important role as a directional cholesterol transporter in the 
Table 3 Differentially expressed genes within GWS QTL regions

\begin{tabular}{|c|c|c|c|c|c|}
\hline Gene & Full name & SSC positions (Sscrofa9) & QTL & Breed $^{* *}$ & DE tissue \\
\hline C6orf211 & chromosome 6 open reading frame 211 & 1: $15,909,144-15,924,522$ & $1 a$ & $\mathrm{D}$ & Testis \\
\hline GPD1L & glycerol-3-phosphate dehydrogenase 1-like & 1: $44,341,710-44,342,765$ & $1 \mathrm{c}$ & L & Testis \\
\hline GAS2 & growth arrest-specific 2 & hsa11: 22,647,188-22,834,601* & $2 a$ & L & Testis \\
\hline DCl & dodecenoyl-CoA isomerase & hsa16: 2,289,396-2,301,615* & $3 a$ & D & Testis \\
\hline GLIS2 & GLIS family zinc finger 2 & hsa16: 4,364,762-4,389,598* & $3 a$ & D & $\begin{array}{l}\text { Liver, } \\
\text { testis }\end{array}$ \\
\hline TIGD7 & tigger transposable element derived 7 & 3: $34,867,636-34,868,997$ & $3 a$ & L & Testis \\
\hline RAB11FIP3 & RAB11 family interacting protein 3 (class II) & 3: $35,607,368-35,662,467$ & $3 a$ & D & Testis \\
\hline NARFL & nuclear prelamin A recognition factor-like & 3: $35,702,474-35,708,696$ & $3 a$ & D & Liver \\
\hline GNPTG & $\mathrm{N}$-acetylglucosamine-1-phosphate transferase, gamma subunit & 3: $36,242,649-36,244,181$ & $3 a$ & L & Testis \\
\hline IL1A & interleukin 1, alpha & 3: $39,059,355-39,072,049$ & $3 a$ & $\mathrm{D}$ & Testis \\
\hline IL1R1 & interleukin 1 receptor, type I & 3: $47,896,450-47,909,993$ & $3 a$ & D & Liver \\
\hline CALB1 & calbindin $1,28 \mathrm{kDa}$ & 4: 47,870,808-47,893,791 & 4 & D & Testis \\
\hline SCUBE3 & signal peptide, CUB domain, EGF-like 3 & $7: 35,596,571-35,617,151$ & $7 a$ & L & Testis \\
\hline C6orf89 & chromosome 6 open reading frame 89 & $7: 37,180,914-37,212,104$ & $7 a$ & D & Testis \\
\hline PTPN9 & protein tyrosine phosphatase, non-receptor type 9 & 7: 63,302,924-63,419,418 & $7 b$ & $\mathrm{D}$ & Testis \\
\hline CYP11A1 & cytochrome P450, family 11, subfamily A, polypeptide 1 & hsa15: 74,630,100-74,660,081* & $7 b$ & $D, L$ & Testis \\
\hline EXOSC8 & exosome component 8 & 11: $12,214,101-12,412,285$ & $11 a$ & D & Testis \\
\hline KCNMB2 & $\begin{array}{l}\text { potassium large conductance calcium-activated channel, subfam M, b memb } \\
2\end{array}$ & 13: $89,166,152-89,194,414$ & $13 b$ & L & Liver \\
\hline MLF1IP & MLF1 interacting protein & 15: 42,999,826-43,027,571 & 15 & D & Testis \\
\hline SORBS2 & sorbin and SH3 domain containing 2 & 15: 43,835,332-44,063,424 & 15 & L & Liver \\
\hline TM2D2 & TM2 domain containing 2 & 15: 44,964,565-44,971,596 & 15 & L & Liver \\
\hline RBPMS & RNA binding protein with multiple splicing & 15: 51,472,724-51,785,193 & 15 & D & Testis \\
\hline FMNL2 & formin-like 2 & 15: $56,320,296-56,433,061$ & 15 & L & Testis \\
\hline ARL6IP6 & ADP-ribosylation-like factor 6 interacting protein 6 & 15: $56,490,709-56,522,096$ & 15 & $L$ & $\begin{array}{l}\text { Liver, } \\
\text { testis }\end{array}$ \\
\hline CD302 & CD302 molecule & $15: 62,141,546-62,172,624$ & 15 & D & Testis \\
\hline GCG & glucagon & 15: $64,064,865-64,074,555$ & 15 & L & Testis \\
\hline RND3 & Rho family GTPase 3 & $\begin{array}{l}\text { hsa2: } 151,324,709- \\
151,395,525^{*}\end{array}$ & 15 & L & Liver \\
\hline STAR & steroidogenic acute regulatory protein & hsa8: $38,000,226-38,008,783^{*}$ & 15 & L & Testis \\
\hline TNFAIP6 & tumor necrosis factor, alpha-induced protein 6 & $\begin{array}{l}\text { hsa2: } 152,214,106- \\
152,236,560^{*}\end{array}$ & 15 & L & Testis \\
\hline ZRANB3 & zinc finger, RAN-binding domain containing 3 & $\begin{array}{l}\text { hsa2: } 135,894,486- \\
136,288,806^{*}\end{array}$ & 15 & $\mathrm{D}$ & Testis \\
\hline
\end{tabular}

Gene expression data was obtained from Moe et al. [20,21].

* Gives the comparative human genomic positions on Build37 for the genes not annotated in Sscrofa9.

** Breeds are $L=$ Norwegian Landrace and $D=$ Norwegian Duroc.

maintenance of cellular cholesterol homeostasis [32], and is required to maintain optimum steroid synthesis [33].

\section{SSC3}

In SSC3 we obtained two GWS QTL regions affecting AndroF and AndroP. QTL3a embraces the region 32.2$53.6 \mathrm{Mb}$ in Landrace and $38-40 \mathrm{Mb}$ in Duroc, while QTL3b was detected in Landrace only and is positioned around $107 \mathrm{Mb}$. Support for QTL3a is provided by Quintanilla et al. [14] who reported a QTL between the markers SW487-S0372 affecting androstenone at different ages in a Large-White $\times$ Meishan cross. We found that the QTL3a is also affecting estrogens in both breeds, and that the Landrace specific QTL3b affects both testosterone (results not shown) and estrogens (Table 1). The genes DCI, GLIS2, TIGD7, RAB11FIP3, NARFL, GNPTG, IL1A and IL1R1 are all localized within QTL3a (Table 3) and found to be differentially expressed in testis or liver in high androstenone Landrace and/or Duroc [20,21]. Interestingly, IL1A has been found to interact with CYP1A1 [34], which is localized within the QTL7b peak (see Table 1). CYP1A1 has previously been proposed as a candidate gene for skatole [35], but is also recently shown to be involved in steroidogenesis [36]. Another candidate in the QTL3a is 


\begin{tabular}{|c|c|c|c|c|c|c|}
\hline SSC & QTL & Breed & LnLikratio & CWS_P** & GWS_P** & Mb_Conf.Int. ${ }^{\$}$ \\
\hline 1 & $1 a^{5}$ & $L^{*} / D$ & $5.56 / 13.0$ & 0.0003 & 0.005 & $165.5-170.1$ \\
\hline 5 & $5^{\mathrm{s}}$ & $L^{*}$ & 11.45 & 0.0007 & 0.013 & $63.2-68.4$ \\
\hline 6 & $6 a^{5}$ & $L^{*}$ & 36.30 & $<0.0001$ & $<0.001$ & $3.7-5.0$ \\
\hline 6 & $6 b^{5}$ & $L^{*}$ & 16.50 & $<0.0001$ & $<0.001$ & $32.2-39.4$ \\
\hline 7 & $7 a^{5}$ & $L / D^{*}$ & 27.98/10.25 & $<0.0001$ & $<0.001$ & $61.5-69.6$ \\
\hline 7 & $7 b^{5}$ & $L / D^{*}$ & $24.29 / 10.25$ & $<0.0001$ & $<0.001$ & 75.9-81.6 \\
\hline 10 & $10 a^{5}$ & $L^{*}$ & 10.83 & 0.0011 & 0.018 & $11.3-13.6$ \\
\hline 10 & $10 b^{5}$ & $L^{*}$ & 14.52 & 0.0001 & 0.002 & $53.1-55.3$ \\
\hline 11 & $11^{\mathrm{s}}$ & $L$ & 9.88 & 0.0017 & 0.030 & $15.7-16.7$ \\
\hline 13 & $13^{5}$ & $D^{*}$ & 10.81 & 0.0010 & 0.018 & $60.5-65.6$ \\
\hline 14 & $14 a^{5}$ & $L^{*}$ & 10.34 & 0.0017 & 0.031 & $38.1-39.1$ \\
\hline 14 & $14 b^{5}$ & $L^{*} / D^{*}$ & $9.76 / 8.27$ & 0.0024 & 0.042 & $146.4-148.4$ \\
\hline
\end{tabular}

* Additionally at least Chromosome Wide Significant for indole

** The highest P-value from the two breeds

${ }^{s}$ All QTLs for skatole are marked with s.

$\$$ The boundaries of the $90 \%$-confidence-interval in mega-bases $(\mathrm{Mb})$.

STARD7, which is involved in cellular cholesterol homeostasis [37].

Using Haploview [38] we detected a highly conserved region (43-45 $\mathrm{Mb}$ ) in QTL3a containing an interesting candidate gene UXS1 (at $44 \mathrm{Mb}$ ). This gene encodes enzymes catalyzing the formation of progesterone, is involved in the formation of UDP-xylose, and influences 3-beta-hydroxy-delta5-steroid dehydrogenase (HSD3B) activity [39]. It is well known in the boar taint research that the HSD3B enzymes are essential for the biosynthesis of all active steroid hormones [40]. Additionally, UXS1 is shown to be differentially expressed after induction of anterior pituitary hormone $(\mathrm{ACTH})$, an important developmental signal that accelerates the appearance of a more mature phenotype in the adrenal glands of young individuals [41].

\section{SSC4}

On SSC4 a QTL for AndroF and AndroP was detected in Duroc only at a position around $48 \mathrm{Mb}$. This QTL is not affecting any of the other sex hormones and might therefore be very interesting for selection purposes. Other QTLs have previously been detected on SSC4, although they are all located to other chromosomal positions $[14,15]$. The DECR1 gene is located within the narrow QTL position detected in the current study. DECR1, a nuclear encoded mitochondrial enzyme that participates in the $\beta$-oxidation pathway, has previously been suggested as a candidate involved in several meat quality traits [42]. Furthermore, the candidate gene CALB1 (around $47.9 \mathrm{Mb}$ ), was found to be differentially expressed in testis from Duroc with extreme high/low levels of androstenone [20]. Interestingly, results suggest that CALB1 is regulated by estrogens [43] and other steroid hormones and is through this probably influencing the sexual development and function [44].

\section{SSC5}

\section{Androstenone}

A highly convincing GWS Duroc specific QTL for AndroF was detected on SSC5 (QTL5a; 20.4-22.2Mb, Figure 3). Comparative genomic alignment to human sequence revealed that the hydroxysteroid (17-beta) dehydrogenase 6 homolog (HSD17B6), which is known to be involved in steroid metabolism [45], is expected to be located within this region. Additionally, the gene encoding retinol dehydrogenase ( $R d h 5)$, which is found to be involved in hydroxysteroid dehydrogenase activity by recognizing 5 alpha-androstan-3alpha, 17beta-diol and androsterone as substrates [46], is expected to be localized within this QTL region. With respect to implementation in breeding schemes, it is very interesting to note that this QTL does not affect any of the other sex related hormones in this study. Furthermore, a Landrace specific QTL (5b) affecting AndroF, AndroP and the other sex hormones was detected in position 32.4-34.9 $\mathrm{Mb}$. No QTL for androstenone have previously been mapped to SSC5.

\section{Skatole}

A QTL affecting Skat and Indo was detected in Landrace in the region 63.2-68.4 $\mathrm{Mb}$ (Table 4). In exactly the same chromosomal region we also obtained GWS for AndroP and Ediol, and CWS for Testo and Esulph (results not shown). This is a rather gene rich region but no obvious candidate genes were found.

\section{SSC6}

\section{Androstenone}

Recently, Duijvesteijn et al. [17] reported a cluster of 31 SNPs on SSC6 (36.9-44.9 Mb) significantly affecting subcutaneous fat androstenone in a Dutch Duroc sireline. Interestingly, in our study a CWS QTL region affecting both AndroF and AndroP was detected between 35.7 and $42.8 \mathrm{Mb}$ in Duroc, but not in Landrace. This QTL does not significantly affect $(\mathrm{P}<0.05)$ the other sex hormones included in this study, although the effect on Esulph is very close to significant. A large block of strong linkage disequilibrium (LD) was detected between 36.9 and $40 \mathrm{Mb}$ in the Dutch population [17], which is noted to be a very gene rich region. In our study we also see a highly extended amount of LD on approximately the same level as the Dutch study in the region between 35.9 and $39.3 \mathrm{Mb}$ (results not shown). However, the most significant haplotype in the QTL region, 13 SNPs in complete LD between $38-39 \mathrm{Mb}$, are explaining only $1.2 \%$ of the total genetic variation. In our study a Duroc specific GWS QTL was located in the vicinity of $119 \mathrm{Mb}$ (Table 1) which affects AndroF 


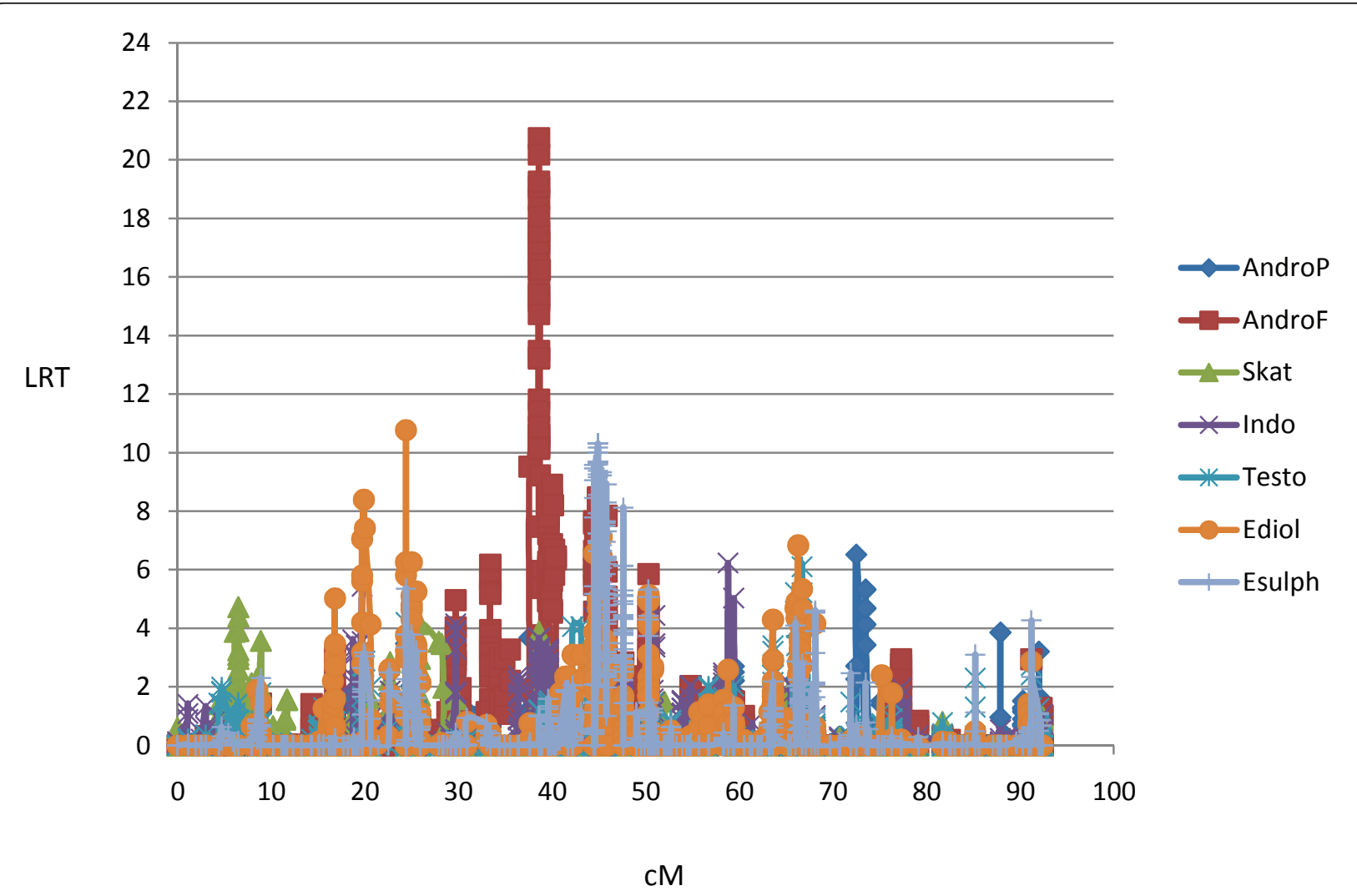

Figure 3 Likelihood ratio (LRT) profile from LDLA for SSC5 in Duroc.

at GWS level and AndroP, Testo, Ediol and Esulph at CWS level.

\section{Skatole}

A Landrace specific QTL for Skat and Indo was obtained on SSC6, region 3.7-5.0 Mb (QTL6a ${ }^{\mathrm{S}}$ ), and demonstrated the highest log-ratio found in this study (Table 4 and Figure 4). Searching for genes in porcine and comparative human genomes using Ensembl failed to reveal any obvious candidates, although several transcription factors are located within this region. Interestingly, a QTL for 'sensory panel skatole' has previously been detected in a Meishan/Large White cross located close to marker SW1353 (approximately position 7.0 $\mathrm{Mb}$ ) in a very low density marker map (Lee et al. [15]). Furthermore, a GWS QTL $\left(6 b^{\mathrm{s}}\right)$ for Skat and Indo is obtained between position 35.9-39.4 Mb (Table 4), which is within the same region as the CWS AndroF QTL described in Duroc above and recently detected by Duijvesteijn et al. [17]. This is a very gene rich region with several biologically relevant genes.

\section{SSC7}

\section{Androstenone}

A large region of highly significant QTL-effects for androstenone was found on SSC7 between positions 33.6 and $88.3 \mathrm{Mb}$ in both breeds. This result is supported by previous studies covering similarly large overlapping QTL regions between markers SW1354 and SW632 [14] and between markers TNFB (position 27.7 Mb in Sscrofa9) and S0066 (position 56.8 Mb in Sscrofa9) [15]. Our results, however, seem to detect three different QTLs within the region, localized at 33.6-41.9 $\mathrm{Mb}$ (QTL7a), 52.8-75.1 Mb (QTL7b) and 80.8-88.3 Mb (QTL7c) (Table 1). Differentiation of these three closely positioned QTLs is made possible by the improved marker density in this study and therefore finer mapping resolution. In addition to the QTLs 7a, 7b and 7c for AndroF, we detect a GWS QTL for AndroP, Testo, Ediol and Esulph (results not shown) in position 27.4-27.9 $\mathrm{Mb}$ which embraces the candidate gene CYP21 (or CYP21A2). CYP21 is a member of the cytochrome P450 superfamily enzymes, which is a key enzyme for corticosteroidogenesis [47] and suggested to have arisen evolutionary from the same gene as CYP17A1 [48]. Physiologically, CYP21 is known to lead to drastic fertility changes in human females [49], and CYP21 has been found to be differentially expressed in Landrace testes with high/low androstenone levels [50]. It is also relevant to note that a QTL for age-at-puberty has been detected in the same region, across a $24 \mathrm{cM}$ interval around marker TNF (position 27.7 Mb in Sscrofa9; [51]), 


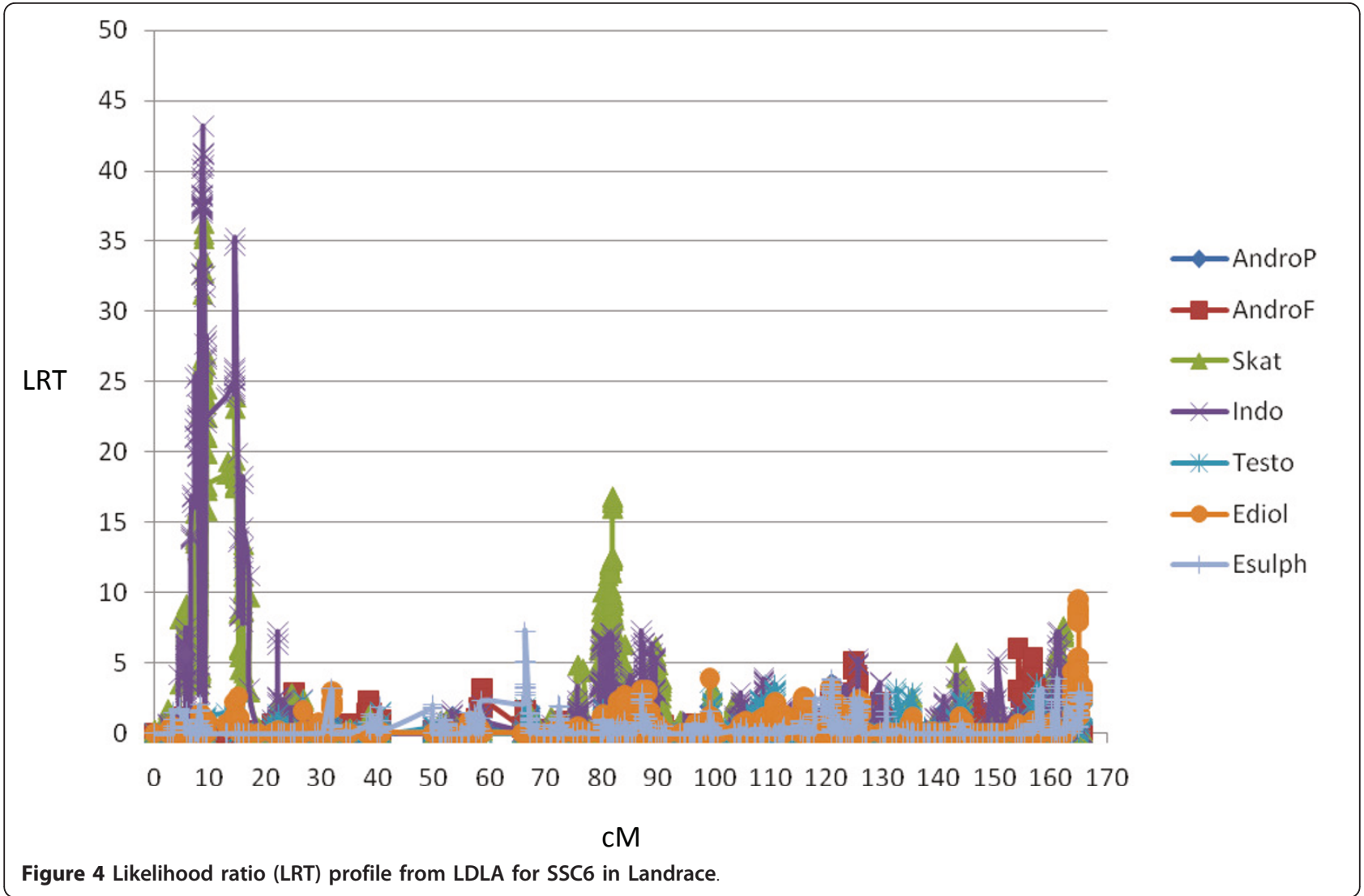

and we know that at least estron sulphate (Esulph) is well known to be correlated with sexual maturity [8]. Two genes located within QTL7a, SCUBE3 and C6orf89 (Table 3 ), are differentially expressed in testis from Landrace and Duroc boars with high/low levels of androstenone, respectively [20]. In addition to being GWS for AndroF and AndroP, and CWS for Testo, Ediol and Esulph, QTL7b is highly GWS for skatole and indole in both breeds (see below). The gene encoding CYP1A1 is located within this region and is involved not only in skatole metabolism [52], but also in steroidogenesis [36]. From comparative mapping with humans, another gene $(C Y P 11 A 1)$ is predicted to be located close to CYP1A1. The CYP11A1 enzyme is known to catalyze the conversion of cholesterol to pregnenolone in the first and rate-limiting step of the steroid hormone synthesis [53]. Previous results from the same Norwegian populations used in this paper show differential expression of CYP11A1 in the liver of high-androstenone boars, however no significant association was detected in Landrace, between a CYP11A1 SNP and androstenone [50]. Similarly, no significant CYP11A1 SNP associations with androstenone were detected in the Large White and Meishan cross performed by Quintanilla et al. [14], which may be evidence to suggest that another candidate gene(s) is involved and explains the differences seen for androstenone level. The gene PTPN9 localized within the QTL7b (Table 3) has previously been found to be upregulated in high androstenone Duroc testis [20]. Only one gene (VRK1; Table 2) has been annotated in QTL7c (126.9-127.5 Mb). This gene has a role in regulating gametogenesis, and disrupted VRK1 function is shown to result in infertile male and female mice [54].

\section{Skatole}

QTL7a $^{\text {s }}$ for Skat is located between 61.5 and $69.6 \mathrm{Mb}$ and therefore overlaps with the QTL7b for androstenone discussed above. The CYP1A1 and CYP1A2 genes are oriented head-to-head in QTL7as and the enzyme products are found to be involved in degradation of skatole $[13,52]$. Additionally, we detected a highly GWS QTL (QTL7b ${ }^{\mathrm{S}}$ ) affecting Skat and Indo in both breeds (Table 4). The LRT profiles for Landrace, covering the most convincing QTL(s), are shown in Figure 5. QTL for skatole have recently been detected on SSC7 in a French population but in a different region [30].

\section{SSC9}

A Landrace specific QTL affecting AndroF on GWS level and AndroP, Ediol and Esulph on CWS level was detected between 7.5 and 8.0 Mb on SSC9. A QTL for androstenone has previously been detected on SSC9 in a 


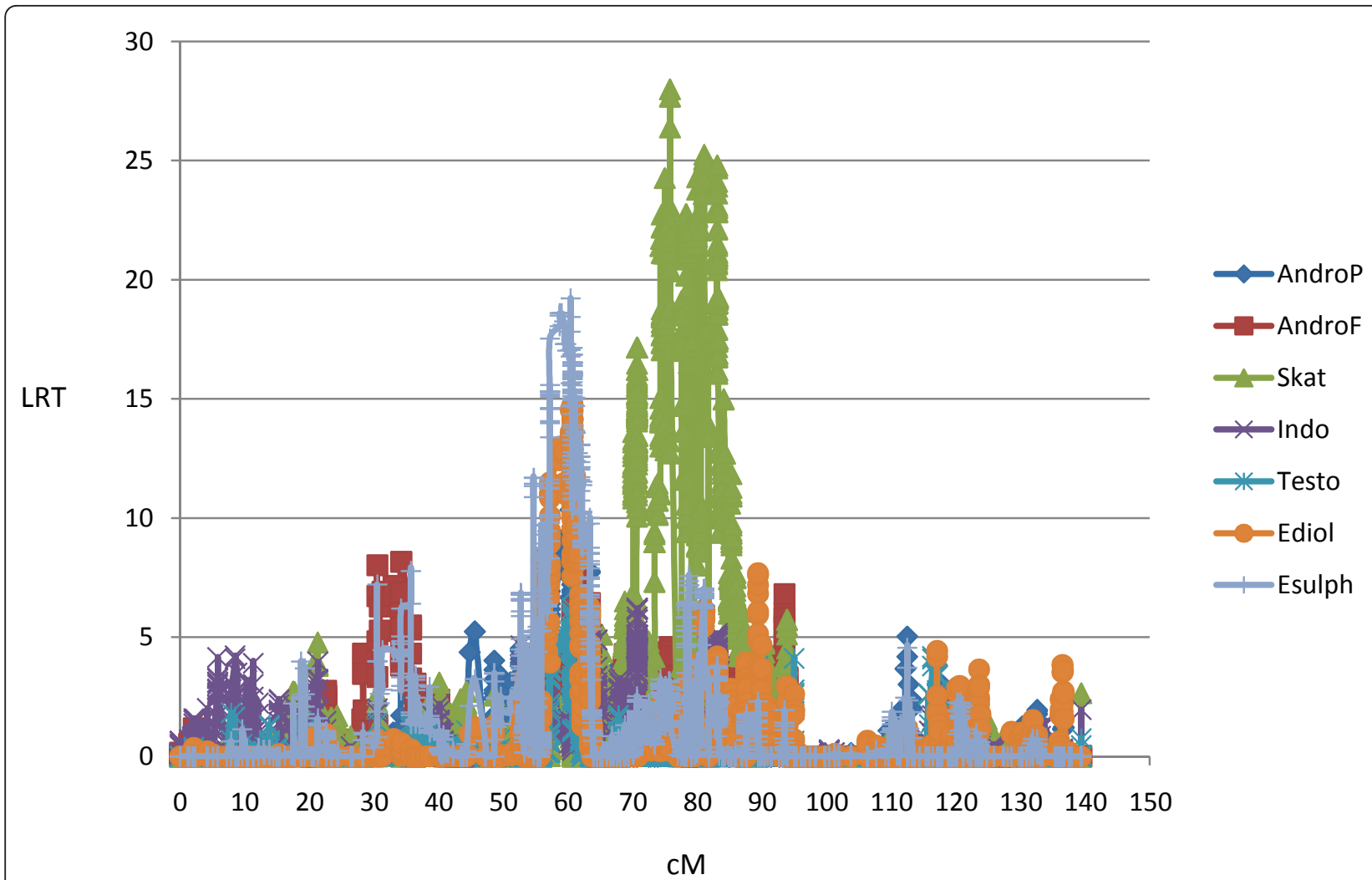

Figure 5 Likelihood ratio (LRT) profile from LDLA for SSC7 in Landrace.

French Large White population [30] and for skatole in a Meishan/Large White cross [15], although these have both been found in different chromosomal regions compared to our study.

\section{SSC10}

\section{Androstenone}

A Duroc specific QTL was detected on SSC10 affecting androstenone at GWS level and affecting Testo, Ediol, Esulph, Skat and Indo at CWS level. No known genes are located within this region in pigs. It is however notable that a QTL for abnormal odor has been reported in the same chromosomal region (between markers SW1041-SW951) by Lee et al. [15].

\section{Skatole}

Two GWS QTLs (QTL10a ${ }^{\mathrm{S}}$ and QTL10b ${ }^{\mathrm{S}}$ ) for Skat and Indo were detected for Landrace only. No other QTL for skatole have previously been detected on SSC10.

\section{SSC11}

\section{Androstenone}

Two GWS breed specific QTLs were detected in positions 11.8-16.7 Mb (QTL11a) and 64.2-64.5 Mb (QTIL11b) in Duroc and Landrace, respectively. Both QTL11a and QTL11b were also at least CWS for both
Ediol and Esulph. A QTL for androstenone in subcutaneous fat has been detected in French Large White [30] but in another position (37.1-37.8 Mb). In the region of QTL11a it was previously found [20] that transcripts from $E X O S C 8$ were differentially expressed in testis from high/low androstenone boars (Table 3).

\section{Skatole}

A Landrace specific QTL was detected in position 15.716.7 Mb affecting Skat but not Indo, in both GWA analysis and LDLA methods. No QTLs for skatole have previously been detected on SSC11.

\section{$\mathrm{SSC} 13$}

\section{Androstenone}

A highly significant and narrow region, 19.3-19.6 Mb (QTL13a) was detected in Duroc for both AndroP and AndroF, as well as for the other three sex steroids investigated in this study (Figure 6). A similar region is detected in Landrace, but for AndroP, Ediol and Esulph only (results not shown). No obvious candidate genes are located within the confidence interval, although two candidate genes in the solute carrier family 22 , members 13 and 14 (SLC22A13 (OAT) and SLC22A14 (OAT1O)) are located very close $(19.1 \mathrm{Mb})$. The substrate panel of SLC22As includes important endogenous compounds 


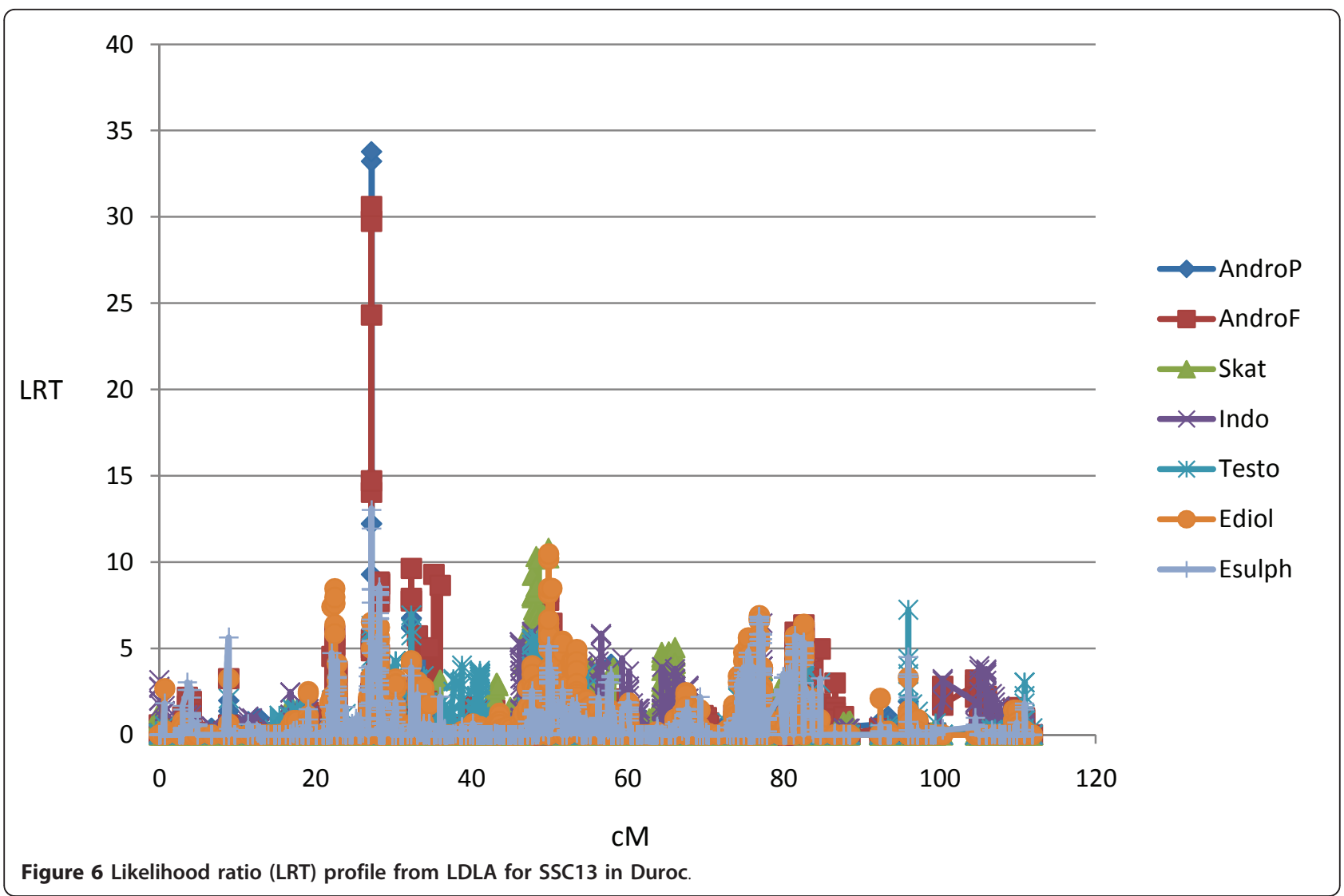

like tryptophan metabolites [55], and sulfated steroids [56]. A Landrace specific QTL for AndroF and the two estrogens was detected from 87.8-92.5 Mb (QTL13b). Although not exactly corresponding, a QTL for 'androstenone at age of 120 days' was detected in a Large White/Meishan cross close to marker SWR1941 at 74 $\mathrm{Mb}$ [14], notably with a much lower marker density. Two other QTLs were detected in a Large White population [30], although at more distant chromosome positions. The gene $K C N M B 2$, expected to be located within QTL13b, was previously found to be differentially expressed in liver of high/low androstenone Landrace boars (Table 3). Interestingly, gene expression of $K C N M B 2$ is previously shown to be increased by $17 \beta-$ estrogen stimulation, although this is studied in human Müller cells only [57].

\section{Skatole}

A Duroc specific QTL for Skat and Indo was detected from 60.5 to $65.6 \mathrm{Mb}$. One QTL has previously been detected for skatole on SSC13 [30] but once again the position is not in agreement with our results.

\section{SSC14}

Two GWS QTLs for skatole were detected on SSC14, one of these was Landrace specific and extended from
38.1 to 39.1 (QTL14a ${ }^{\mathrm{S}}$ ). No QTL for skatole have previously been observed in this region, although a QTL for 'androstenone at: 100, 120, 140 and 160 days' has been detected in the same region in a Large White/Meishan cross [14]. The second QTL extended between 146.6 and 148.4 $\mathrm{Mb}\left(\mathrm{QTL} 14 \mathrm{~b}^{\mathrm{S}}\right)$ and presented GWS for skatole in Landrace, and CWS ( $<0.05)$ in Duroc. Furthermore, the QTL is highly GWS for indole in both breeds. The gene encoding cytochrome P450 2E1 (CYP2E1), which has a central role in the metabolism of skatole [e.g. 58 , 59], is located at $148.1 \mathrm{Mb}$ and is an excellent positional and biological candidate gene for this QTL. A previous study from our group utilizing exactly the same animal material, detected a haplotype within CYP2E1 which explained up to $6 \%$ of the phenotypic variance for skatole and up to $12 \%$ of the phenotypic variance for the indole [60]. Our results are in close agreement with previous findings in Danish Landrace [61].

\section{SSC15}

A very wide GWS QTL located across a confidence interval from 42.5-70.7 $\mathrm{Mb}$ on SSC15 in both breeds was affecting all the traits in this study (at least CWS), except for Indo. This is a very gene rich region including several genes involved in sulfotransferase activity 
belonging to the cytochrome P450 family. From the studies of Moe et al. [20,21] as many as 12 genes within this QTL region were found to be differentially expressed in liver and/or testis in high/low androstenone boars (Table 3). This indicates that several genes might be causing the QTL effect although only the genes encoding GCG (glucagon) and STAR (steroidogenic acute regulatory protein) are known to be correlated with level of different steroids like androgens and estrogens $[50,62,63]$. As far as we know no QTLs for boar taint have previously been found on SSC15.

\section{SSC18}

Two Landrace specific QTLs were detected on SSC18 in positions 13.4-14.2 Mb (QTL18a) and 39.7-40.4 Mb (QTL18b). As far as we know no QTLs for boar taint have previously been detected on SSC18.

\section{Conclusions}

This is the first report utilizing a high density marker map for simultaneous analysis of boar taint compounds and related sex hormones, using both GWA and LDLA approach. Several QTLs appear to be involved in regulation of androstenone and skatole, individually they explain relatively little of the total genetic variation. Most of the QTLs for androstenone are affecting both androstenone and estrogens, making practical implementation in breeding challenging. Most QTLs are also breed specific and we conclude that panels of SNPs for selection need to be designed for each breed separately. However, 7 QTLs were detected in one breed and confirmed in the other breed, i.e. an independent sample. QTLs that were not confirmed across breeds cannot be dismissed since the QTL may not be segregating in the other breed, i.e. the QTL is breed specific, or its effect may be substantially reduced in a different genetic background. QTLs for skatole are mostly not negatively affecting sex hormones and should be easier to use directly into the current breeding scheme. Generally, the combination of high density SNP genotyping using the $60 \mathrm{~K}$ porcine SNP array, GWA and LDLA analysis revealed many genome-wide significant QTLs (25 in total), many of which were confirmed across breeds and tissues where the traits were recorded, and/or method of analysis. Therefore, with the new and more precise information on SNP-effects for androstenone and skatole, we currently suggest a breed specific panel for SNPs affecting androstenone only (not estrogens) and skatole. For further research, more knowledge is needed to determine the relationship between boar- and sow fertility, sex steroids and boar taint compounds to go to the next step of selection using all the genetic markers for androstenone detected in this study. It is a possibility that the effect of the QTLs on the other steroids will reduce boar- and/or sow-fertility, in which case it is a problem for pork production.

\section{Additional material}

Additional file 1: Summary of the genetic maps, SSC1-SSC18, in Landrace and Duroc. An overview of the lengths of all the autosomal chromosomes (SSCs) in basepairs (bp) and centimorgans (CM), as well as number of SNPs per chromosome in Norwegian Landrace and Duroc.

\section{Acknowledgements}

The work was financed by the Norwegian pig breeders association (NORSVIN) and the Research Council of Norway. We want to thank Ellen Dahl and Øystein Andresen for being in charge of analyses (androstenone in subcutaneous fat and plasma, skatole and indole) done at the hormone laboratory at the Norwegian School of Veterinary Science (NVH), and Peter Torjesen for analyses (testosterone, $17 \beta$-estradiol and estron sulphate) performed at the hormone laboratory at Aker University Hospital. We also highly appreciate the contribution from Elin Bergseth at NORSVIN/BioBank AS for collection and handling of samples, Thomas Moen, Harald Grove and Torfinn Nome for helping out with programs for data handling, and Martien Groenen for kindly providing the physical SNP positions of the $60 \mathrm{~K}$ Porcine SNP array.

\section{Author details}

${ }^{1}$ NORSVIN (The Norwegian Pig Breeders Association), PO Box 504, 2304 Hamar, Norway. ${ }^{2}$ Department of Animal and Aquacultural Sciences, Norwegian University of Life Sciences, PO Box 5003, 1432 Ås, Norway. ${ }^{3}$ Centre for Integrative Genetics (CIGENE), Norwegian University of Life Sciences, PO Box 5003 Ås, Norway.

\section{Authors' contributions}

EG was coordinating the study, designing the experiment, organizing sample collection, preparation and genotyping experiment, involved in some of the statistical analyses, and drafted the paper. SL was involved in planning the project, provided laboratory facilities and took part in writing the paper. $\mathrm{HH}$ and $\mathrm{MHSH}$ were performing the preparation of samples, genotyping and quality control of genotypes. MK was responsible for the genotyping facilities, supervising the quality control, and involved in writing the paper. MVS was combining the LDLA and GWA results with the previous gene expression results performed in the same population, involved in discussions and contributed to the paper. THEM was involved in designing the experiment, conducting the statistical analysis and took part in writing the paper. All authors have read and approved the final manuscript.

Received: 18 April 2011 Accepted: 13 July 2011 Published: 13 July 2011

\section{References}

1. Babol J, Squires EJ: Quality of meat from entire male pigs. Food Research International 1995, 28(3):201-212.

2. Dijksterhuis GB, Engel B, Walstra P, Font i Furnols M, Agerhem $H$, Fischer $K$, Oliver MA, Claudi-Magnussen C, Siret F, Béague MP, Homer DB, Bonneau M: An international study on the importance of androstenone and skatole for boar taint: II. Sensory evaluation by trained panels in seven European countries. Meat Science 2000, 54(3):261-269.

3. Grindflek E, Meuwissen T, Aasmundstad T, Hamland H, Hansen M, Nome T, Kent $\mathrm{M}$, Torjesen $\mathrm{P}$, Lien S: Revealing genetic relationships between compounds affecting boar taint and reproduction in pigs. J Anim Sci 2011, 89(3):680-692

4. Sellier P, Le Roy P, Fouilloux MN, Gruand J, Bonneau M: Responses to restricted index selection and genetic parameters for fat androstenone level and sexual maturity status of young boars. Livstestock Production Science 2000, 63:265-274.

5. Gower DB: 16-Unsaturated C 19 steroids. A review of their chemistry, biochemistry and possible physiological role. Journal of Steroid Biochemistry 1972, 3:45-103. 
6. Hess RA: Estrogen in the adult male reproductive tract: a review. Reprod Biol Endocrinol 2003, 1:52.

7. Zamaratskaia G, Madej A, Babol J, Squires E, Lundstrom K: Free oestrone in adipose tissue and its relation to androstenone and skatole in entire male pigs. Reprod Domest Anim 2005, 40(2):156-160.

8. Zamaratskaia G, Rydhmer L, Chen G, Madej A, Andersson HK, Lundstrom K: Boar taint is related to endocrine and anatomical changes at puberty but not to aggressive behaviour in entire male pigs. Reprod Domest Anim 2005, 40(6):500-506.

9. Walker WH: Non-classical actions of testosterone and spermatogenesis. Philos Trans R Soc Lond B Biol Sci 2010, 365(1546):1557-1569.

10. Yokoyama MT, Carlson JR: Microbial metabolites of tryptophan in the intestinal tract with special reference to skatole. Am J Clin Nutr 1979, 32(1):173-178.

11. Lundström K, Malmfors B, Malmfors G, Petersson H, Stern S, Mortensen A, Sorensen S: Boar taint and bitter taste as affected by androstenone and skatole. 30th Eur Meeting of Meat Res Workers Bristol Proc 30th Eur Meeting of Meat Res Workers 1984, 379-398.

12. Vold E: Fleischproduktionseigenschaften bei ebern und kastraten. Meldinger fra Norges Landbrukshøgskole 1970, 49:1-25.

13. Rasmussen MK, Zamaratskaia G, Ekstrand B: In vivo effect of dried chicory root (Cichorium intybus L.) on xenobiotica metabolising cytochrome P450 enzymes in porcine liver. Toxicol Lett 2010.

14. Quintanilla R, Demeure O, Bidanel JP, Milan D, lannuccelli N, Amigues $Y$, Gruand J, Renard C, Chevalet C, Bonneau M: Detection of quantitative trait loci for fat androstenone levels in pigs. J Anim Sci 2003, 81(2):385-394.

15. Lee GJ, Archibald AL, Law AS, Lloyd S, Wood J, Haley CS: Detection of quantitative trait loci for androstenone, skatole and boar taint in a cross between Large White and Meishan pigs. Anim Genet 2005, 36(1):14-22.

16. Varona L, Vidal O, Quintanilla R, Gil M, Sánchez A, Folch J, Hortos M, Rius M, Amills M, Noguera J: Bayesian analysis of quantitative trait loci for boar taint in a Landrace outbred population. J Anim Sci 2005, 83(2):301-307.

17. Duijvesteijn N, Knol EF, Merks JW, Crooijmans RP, Groenen MA, Bovenhuis $\mathrm{H}$, Harlizius B: A genome-wide association study on androstenone levels in pigs reveals a cluster of candidate genes on chromosome 6. BMC Genet 2010, 11(1):42

18. Ramos AM, Crooijmans R, Affara NA, Amaral AJ, Archibald AL, Beever JE, Bendixen C, Churcher C, Clark R, Dehais P, Hansen MS, Hedegaard J, Hu ZL, Kerstens HH, Law AS, Megens HJ, Milan D, Nonneman DJ, Rohrer GA, Rothschild MF, Smith TPL, Schnabel RD, Van Tassell CP, Taylor JF, Wiedmann RT, Schook LB, Groenen MAM: Design of a High Density SNP Genotyping Assay in the Pig Using SNPs Identified and Characterized by Next Generation Sequencing Technology. PLoS One 2009, 4(8).

19. Gregersen V, Panitz F, Vingborg R, Sorensen $K$, Stengaard H, Buije Z, Hoj A, Grindflek E, Moe M, Lien S, Archibald A, Hastings N, Humphrey S, Rogers J, Dunham A, Bendixen C: Performance of a Genome-Wide 7 K Porcine SNP Chip. The 3rd International Symposium on Animal Functional Genomics: 7-9 April 2008; Edinburgh 2008

20. Moe M, Meuwissen T, Lien S, Bendixen C, Wang X, Conley LN, Berget I, Tajet H, Grindflek E: Gene expression profiles in testis of pigs with extreme high and low levels of androstenone. BMC Genomics 2007, 8:405.

21. Moe M, Lien S, Bendixen C, Hedegaard J, Hornshoj H, Berget I, Meuwissen TH, Grindflek E: Gene expression profiles in liver of pigs with extreme high and low levels of androstenone. BMC Vet Res 2008, 4:29.

22. Tuomola M, Harpio R, Knuuttila P, Mikola H, Løvgren T: Time-resolved fluoroimmunoassay for the measurement of androstenone in porcine serum and fat samples. Journal of agricultural food chemistry 1997, 45:3529-3534.

23. Andresen Ø: Development of radioimunoassay for 5alpha-adrost-16-en-3one in pig peripheral plasma. Acta endochrinologia 1974, 76:377-387.

24. Tuomola M, Vahva M, Kallio H: High-Performance liquid chromatography determination of skatole and indole levels in pig serum, subcutaneous fat and submaxillary salivary glands. Journal of agricultur food chemistry 1996, 44:1265-1270.

25. Green P, Falls K, Crooks S: Documentation for CRI-MAP, version 2.4 . Washington University School of Medicine, St Louis, MO 1990.

26. Meuwissen TH, Goddard ME: Multipoint identity-by-descent prediction using dense markers to map quantitative trait loci and estimate effective population size. Genetics 2007, 176(4):2551-2560.

27. Patterson $\mathrm{H}$, Thompson R: Recovery of inter-block information when block sizes are unequal. Biometrika 1971, 58(3):545-554.
28. Piepho HP: A quick method for computing approximate thresholds for quantitative trait loci detection. Genetics 2001, 157(1):425-432.

29. R-Development-Core-Team: R:A Language and Environment for Statistical Computing. R Foundation for Statistical Computing 2011 [http://www.Rproject.org].

30. Le Mignon G, lannuccelli N, Robic A, Billon Y, Bidanel JP, Larzul C: Fine Mapping Of Quantitative Trait Loci For Androstenone And Skatole Levels In Pig. 9th WCGALP, 1-6 August, Leipzig, Germany 2010.

31. De Fabiani E, Mitro N, Gilardi F, Crestani M: Sterol-protein interactions in cholesterol and bile acid synthesis. Subcell Biochem 2010, 51:109-135.

32. Rodriguez-Agudo D, Ren S, Wong E, Marques D, Redford K, Gil G, Hylemon P, Pandak WM: Intracellular cholesterol transporter StarD4 binds free cholesterol and increases cholesteryl ester formation. I Lipid Res 2008, 49(7):1409-1419.

33. Hu J, Zhang Z, Shen WJ, Azhar S: Cellular cholesterol delivery, intracellular processing and utilization for biosynthesis of steroid hormones. Nutr Metab (Lond) 2010, 7:47.

34. Gumus ZH, Du B, Kacker A, Boyle JO, Bocker JM, Mukherjee P, Subbaramaiah K, Dannenberg AJ, Weinstein H: Effects of tobacco smoke on gene expression and cellular pathways in a cellular model of oral leukoplakia. Cancer Prev Res (Phila) 2008, 1(2):100-111.

35. Diaz GJ, Squires EJ: Metabolism of 3-methylindole by porcine liver microsomes: responsible cytochrome P450 enzymes. Toxicol Sci 2000, 55(2):284-292.

36. Fan J, Traore K, Li W, Amri H, Huang H, Wu C, Chen H, Zirkin B, Papadopoulos V: Molecular mechanisms mediating the effect of mono(2-ethylhexyl) phthalate on hormone-stimulated steroidogenesis in MA10 mouse tumor Leydig cells. Endocrinology 2010, 151(7):3348-3362.

37. Horibata Y, Sugimoto H: StarD7 mediates the intracellular trafficking of phosphatidylcholine to mitochondria. J Biol Chem 2010, 285(10):7358-7365

38. Barrett JC, Fry B, Maller J, Daly MJ: Haploview: analysis and visualization of LD and haplotype maps. Bioinformatics 2005, 21(2):263-265.

39. Bray JE, Marsden BD, Oppermann U: The human short-chain dehydrogenase/reductase (SDR) superfamily: a bioinformatics summary. Chem Biol Interact 2009, 178(1-3):99-109.

40. Payne AH, Hales DB: Overview of steroidogenic enzymes in the pathway from cholesterol to active steroid hormones. Endocr Rev 2004, 25(6):947-970

41. Lee JJ, Widmaier EP: Gene array analysis of the effects of chronic adrenocorticotropic hormone in vivo on immature rat adrenal glands. J Steroid Biochem Mol Biol 2005, 96(1):31-44.

42. Amills $M$, Vidal O, Varona L, Tomas A, Gil M, Sanchez A, Noguera JL: Polymorphism of the pig 2,4-dienoyl CoA reductase 1 gene (DECR1) and its association with carcass and meat quality traits. J Anim Sci 2005, 83(3):493-498.

43. Opperman LA, Saunders TJ, Bruns DE, Boyd JC, Mills SE, Bruns ME: Estrogen inhibits calbindin-D28k expression in mouse uterus. Endocrinology 1992, 130(3):1728-1735.

44. Stuart EB, Thompson JM, Rhees RW, Lephart ED: Steroid hormone influence on brain calbindin- $D(28 \mathrm{~K})$ in male prepubertal and ovariectomized rats. Brain Res Dev Brain Res 2001, 129(2):125-133.

45. Moeller $\mathrm{G}$, Adamski J: Multifunctionality of human 17beta-hydroxysteroid dehydrogenases. Mol Cell Endocrinol 2006, 248(1-2):47-55.

46. Wang J, Chai X, Eriksson U, Napoli JL: Activity of human 11-cis-retinol dehydrogenase (Rdh5) with steroids and retinoids and expression of its mRNA in extra-ocular human tissue. Biochem J 1999, 338(Pt 1):23-27.

47. Crawford RJ, Hammond VE, Connell JM, Coghlan JP: The structure and activity of two cytochrome P450c21 proteins encoded in the ovine adrenal cortex. J Biol Chem 1992, 267(23):16212-16218.

48. Picado-Leonard J, Miller WL: Cloning and sequence of the human gene for P450c17 (steroid 17 alpha-hydroxylase/17,20 lyase): similarity with the gene for P450c21. DNA 1987, 6(5):439-448.

49. New Ml: Steroid 21-hydroxylase deficiency (congenital adrenal hyperplasia). Am J Med 1995, 98(1A):2S-8S.

50. Grindflek E, Berget I, Moe M, Oeth P, Lien S: Transcript profiling of candidate genes in testis of pigs exhibiting large differences in androstenone levels. BMC Genet 2010, 11:4.

51. Cassady JP, Johnson RK, Pomp D, Rohrer GA, Van Vleck LD, Spiegel EK, Gilson KM: Identification of quantitative trait loci affecting reproduction in pigs. J Anim Sci 2001, 79(3):623-633. 
52. Lanza D, Yost G: Selective dehydrogenation/oxygenation of 3methylindole by cytochrome P450 enzymes. Drug Metabolism and Disposition 2001, 29(7):950-953.

53. Miller WL: Mitochondrial specificity of the early steps in steroidogenesis. J Steroid Biochem Mol Biol 1995, 55(5-6):607-616.

54. Wiebe MS, Nichols RJ, Molitor TP, Lindgren JK, Traktman P: Mice deficient in the serine/threonine protein kinase VRK1 are infertile due to a progressive loss of spermatogonia. Biol Reprod 2010, 82(1):182-193.

55. Bahn A, Ljubojevic M, Lorenz H, Schultz C, Ghebremedhin E, Ugele B, Sabolic I, Burckhardt G, Hagos Y: Murine renal organic anion transporters mOAT1 and mOAT3 facilitate the transport of neuroactive tryptophan metabolites. Am J Physiol-Cell Physiol 2005, 289(5):C1075-C1084.

56. Burckhardt BC, Burckhardt G: Transport of organic anions across the basolateral membrane of proximal tubule cells. Rev Physiol Biochem Pharmacol 2003, 146:95-158.

57. Li C, Tang Y, Li F, Turner S, Li K, Zhou X, Centola M, Yan X, Cao W: 17betaestradiol (betaE2) protects human retinal Muller cell against oxidative stress in vitro: evaluation of its effects on gene expression by cDNA microarray. Glia 2006, 53(4):392-400.

58. Babol J, Squires E, Lundström K: Hepatic metabolism of skatole in pigs by cytochrome P4502E1. J Anim Sci 1998, 76(3):822-828.

59. Skinner T, Doran E, McGivan J, Haley C, Archibald A: Cloning and mapping of the porcine cytochrome-p450 2E1 gene and its association with skatole levels in the domestic pig. Animal Genetics 2005, 36(5):417-422.

60. Moe M, Lien S, Aasmundstad T, Meuwissen THE, Hansen MHS, Bendixen C, Grindflek E: Association between SNPs within candidate genes and compounds related to boar taint and reproduction. BMC Genetics 2009, 10(32).

61. Karacaören B, de Koning DJ, Velander I, Petersen S, Haley CS, Archibald AL: Alternative Association Analyses on Boar Taint using Discordant Sib Pairs Experimental Design. Proc 60th EAAP, Barcelona, Spain 2009.

62. Christoffersen BO, Gade LP, Golozoubova V, Svendsen O, Raun K: Influence of castration-induced testosterone and estradiol deficiency on obesity and glucose metabolism in male Gottingen minipigs. Steroids 2010, 75(10):676-684.

63. Christenson LK, Strauss JF: Steroidogenic acute regulatory protein (StAR) and the intramitochondrial translocation of cholesterol. Biochim Biophys Acta 2000, 1529(1-3):175-187.

doi:10.1186/1471-2164-12-362

Cite this article as: Grindflek et al:: Large scale genome-wide association and LDLA mapping study identifies QTLs for boar taint and related sex steroids. BMC Genomics 2011 12:362.

\section{Submit your next manuscript to BioMed Central and take full advantage of:}

- Convenient online submission

- Thorough peer review

- No space constraints or color figure charges

- Immediate publication on acceptance

- Inclusion in PubMed, CAS, Scopus and Google Scholar

- Research which is freely available for redistribution 